Research Article

\title{
Cognitive Emotional Regulation Model in Human-Robot Interaction
}

\author{
Xin Liu, ${ }^{1}$ Lun Xie, ${ }^{1}$ Anqi Liu, ${ }^{2}$ and Dan $\mathrm{Li}^{1}$ \\ ${ }^{1}$ School of Computer and Communication Engineering, University of Science and Technology Beijing, Beijing 100083, China \\ ${ }^{2}$ Beijing Shougang International Engineering Technology Limited Company, Beijing 100043, China
}

Correspondence should be addressed to Lun Xie; xielun@ustb.edu.cn

Received 23 September 2014; Revised 20 December 2014; Accepted 21 December 2014

Academic Editor: Qingang Xiong

Copyright (C) 2015 Xin Liu et al. This is an open access article distributed under the Creative Commons Attribution License, which permits unrestricted use, distribution, and reproduction in any medium, provided the original work is properly cited.

This paper integrated Gross cognitive process into the HMM (hidden Markov model) emotional regulation method and implemented human-robot emotional interaction with facial expressions and behaviors. Here, energy was the psychological driving force of emotional transition in the cognitive emotional model. The input facial expression was translated into external energy by expression-emotion mapping. Robot's next emotional state was determined by the cognitive energy (the stimulus after cognition) and its own current emotional energy's size and source's position. The two random quantities in emotional transition process-the emotional family and the specific emotional state in the AVS (arousal-valence-stance) 3D space-were used to simulate human emotion selection. The model had been verified by an emotional robot with 10 degrees of freedom and more than 100 kinds of facial expressions. Experimental results show that the emotional regulation model does not simply provide the typical classification and jump in terms of a set of emotional labels but that it operates in a 3D emotional space enabling a wide range of intermediary emotional states to be obtained. So the robot with cognitive emotional regulation model is more intelligent and real; moreover it can give full play to its emotional diversification in the interaction.

\section{Introduction}

Nowadays, robot not only needs intelligent behavior but also needs mental life, such as cognition, emotion, and personality. Robot evolves its own emotional intelligence for anthropomorphic and diversified states and even produces empathy. Human-robot interaction requires emotional analysis and regulation, so emotional modeling is particularly important. In this section, several valued and far-reaching approaches about emotion modeling have been proposed. They can be divided into two categories: the discrete model and the emotional space model. Moreover, the two categories are independent and complement.

1.1. The Discrete Model. Izzard divides emotions into two categories: prototypical emotions and complex emotions. The prototypical emotions include more or less discrete emotional states, usually from 2 to 11 [1-3]. Lazarus believes that the growing importance of cognitive-mediational or value-expectancy approaches to mind and behavior in social sciences has promoted the prosperity of emotions as discrete categories [4]. Ekman proposes six prototypical emotions based on the facial expressions [5]. Besides, this analogous approach is followed by several authors. The artificial emotions are divided into anger, boredom, fear, happiness, interest, and sadness in Cañamero's works with social robots [6]. In Gadanho's approach, emotions (happiness, fear, sadness, and anger) are related to certain events [7]. Velásquez also proposes an emotion-based control for autonomous robots. In his research, six prototypical emotions (anger, fear, sorrow, happiness, disgust, and surprise) are implemented with innate personality and the capacity of acquired learning [8]. Murphy et al. put forward the artificial emotional states (happy, confident, concerned, and frustrated) for multiagent systems, and the emotions are released depending on the task process [9]. Complex emotions consist of the following three categories: (1) 2-3 prototypical emotions mixed; (2) prototypical emotions and inner impulse mixed; (3) prototypical emotions and affective-cognitive structure mixed [10]. 
1.2. The Emotional Space Model. Unlike discrete models, the emotional space models consider a continuous multidimensional space where each point stands for an emotional state and each dimension stands for a fundamental property common to all emotions. In order to fully show the emotional properties, the classical 3D emotional space, pleasant/unpleasant, excitement/depression, and tension/relaxation, has already been used by Wundt [11]. For many years, a large number of emotional dimensional theories have been proposed [12-15]. One of the most accepted theories is described by Russell and Mehrabian [16]: pleasurearousal-dominance space (PAD). Scherer and Ekam and Ortony et al. also use and develop PAD space to determine artificial emotions for social robots [17, 18]. Zecca et al. construct the 3D psychological vector space, arousalpleasant-certain, with machine learning, dynamic regulation, and personality [19]. In addition, Breazeal's arousal-valencestance (AVS) space with the social robot, Kismet, is the most noteworthy emotional space model [20]. In that case, the emotional state of the robot varies according to its interaction with people. In fact, the external stimulus is labeled by three emotional properties in the AVS space. Ortony et al. model the OCC reasoning process that can produce the cognitive emotions and touch off complex emotional experiences via the trend of events (including event, object, and agent) [18]. Then this model has been developed and refined by Yang et al. and Kim et al. [21, 22].

This paper discussed a cognitive emotional regulation model in the active field state space. First, based on Gross cognitive strategy, the cognitive reappraisal model satisfied Fischna-Weber law was built, and a physiological endurance coefficient was defined to meet the psychological diversity. Second, emotional state space in the active field was established on the solid basis of the physiological energy distribution, and the transition probability among the emotional families was figured out. Third, an observational state sequence was yielded from emotional family by HMM. Finally, the emotional regulation model is devoted to humanrobot experiment for the validity analysis. Figure 1 gives out general ideas of this research.

The rest of this paper is organized as follows. Section 2 discusses the Gross cognitive strategy and presents a cognitive reappraisal model. Section 3 defines the emotional space and related state transition process. Section 4 introduces the emotional robot platform. Section 5 shows experimental results and discussions. The conclusion and directions for future work are offered in Section 6.

\section{Emotional Cognition and Modeling}

2.1. Gross Cognitive Strategy. Gross proposed five emotional regulation strategies-situation selection, situation modification, attention deployment, cognitive reappraisal, and response suppression [23]. Cognitive reappraisal, the foremost antecedent-focused strategy in the early emotional regulation stage, depends on the internal willpower and personality factors. When the individual is in troubles or psychological expectation is different to the reality, the negative

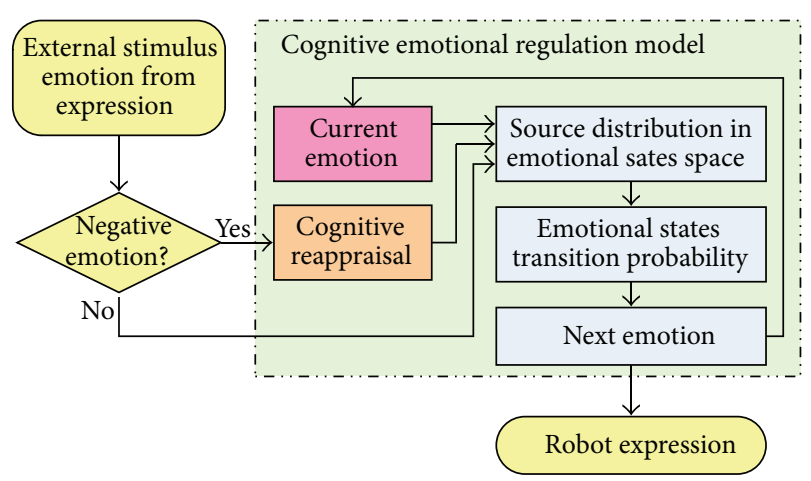

FIgURE 1: General ideas of this research.

emotions, such as sadness, anxiety, anger, and pain, are produced. In general, the cognitive reappraisal can well cut down the negative emotional intensity and improve the individual emotional experience. In the cognitive process cognitive reappraisal and response suppression strategies both can improve the individual emotional experience. However, the psychological research has proven that the cognitive reappraisal strategy, as compared with the four other regulation strategies, is most effective in the negative emotional regulation.

2.2. Cognitive Reappraisal Model. Gross believes that spontaneity cognitive reappraisal, as an essential part of psychological defense mechanism, could help individuals to consider the emotional stimulus from the peaceful perspective [23]. Thus, facing various external emotional stimulus, psychological system will first unconsciously start the spontaneous cognitive reappraisal to reduce the emotional intensity. In other words, when the external stimulus appears in emotional state space, the spontaneous cognitive reappraisal comes into play at once for cutting down the stimulusintensity. Because the spontaneous cognitive reappraisal mainly depends on individual physiological endurance, a physiological endurance coefficient $\chi \in(0,1)$ is defined for robot.

Fischna-Weber law is a psychophysical formula describing the relationship of internal feel-intensity and external stimulus-intensity. The law shows that human feel-intensity is proportional to the logarithm of stimulus-intensity (shown in formula (1)). Under the spontaneity guidance cognitive reappraisal, the feel-intensity of the negative emotion can be drawn from formula (2):

$$
\begin{aligned}
& \mu=K m \log I+C, \\
& \mu^{\prime}=\chi(K m \log I+C) .
\end{aligned}
$$

Here, $\mu$ is the feel-intensity before spontaneity cognitive reappraisal, $\mu^{\prime}$ is the feel-intensity after spontaneity cognitive reappraisal, $I$ is the stimulus-intensity, and $K m$ and $C$ are constant.

When $\chi \rightarrow 0$, robot has better endurance and enhances the defense capability of external negative emotional stimuli. 


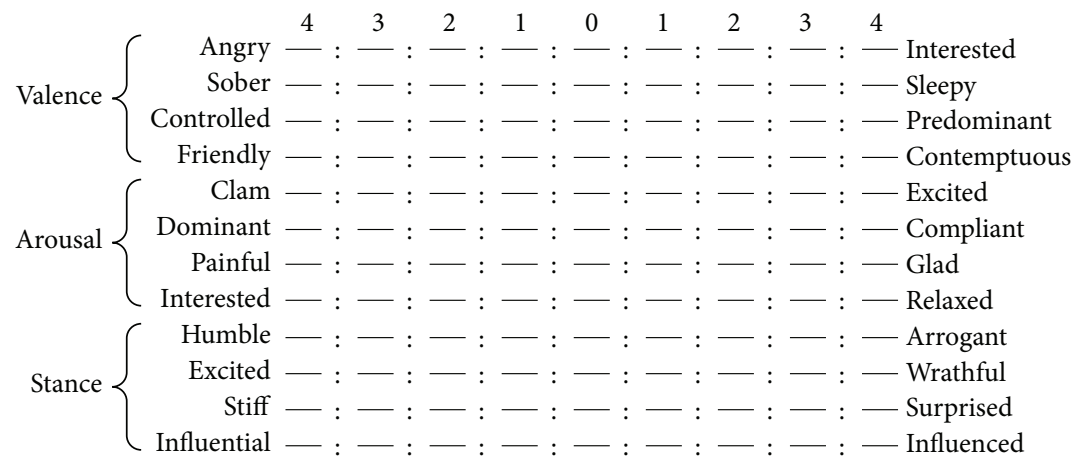

FIgURE 2: A simplified version of the affect scale.

Conversely, when $\chi \rightarrow 1$, robot's endurance declines and its own emotion state is vulnerable to external impacts.

Based on AVS emotional model, a simplified version of the affect scale is designed. In this scale, each dimensionality (arousal, valence, and stance) is measured by four pairs of emotional states, as is shown in Figure 2. Each pair includes two opposite emotional states divided into 9 levels in a dimension, and the two states are fundamentally the same in the other two dimensions. There are 7 typical experiment scenes corresponding to 7 external stimulus emotions and 500 volunteers of random selection in different ages including five groups of 11 20, 21 30, 31 40, 41 50, and 51 60 years. Groups of 100 participants get external stimulus emotion and choose emotional level in the scale at once and after thinking a while, respectively. By processing and analysis of each scene's data with the hypothesis test based on Gaussian iteration, we can find that after the cognitive reappraisal the influence of positive scenes has no obvious change and the change of negative emotion obeys Gauss distribution shown in Figure 3. In this hypothesis test, we first calculate the Gauss mathematical expectation $\widehat{\mu}$ for each scene by maximum likelihood estimation. Calculate the distance between each piece of data and the center of Gauss distribution and delete the date which has the max distance. Then calculate the new mathematical expectation $\widehat{\mu}^{\prime}$ by maximum likelihood and obtain the distance $\left|\widehat{\mu}^{\prime}-\widehat{\mu}\right|$. If the distance $\left|\widehat{\mu}^{\prime}-\widehat{\mu}\right|$ is less than the threshold, the iteration will finish. If the algorithm converges quickly, the AVS data for this scene is subject to Gaussian distribution. So the parameter $\chi$, a set of Gauss random data, could imitate human spontaneity cognition and make robot have a probabilistic and diversified cognitive style.

\section{Emotional Regulation Modeling}

3.1. Spatial Description of Emotional State. The state space of robot's emotions $S$, where emotions can transform freely, is a set of robot's all emotions. Robot's own emotional state $S_{t}$ can be considered as a spatial location at time $t$ in the active field state space. And each state has the corresponding psychology energy, which is determined by the potential energy in the active field state space. Different to traditional limited emotional states, emotional regulation process is defined in the case of continuous time and continuous state

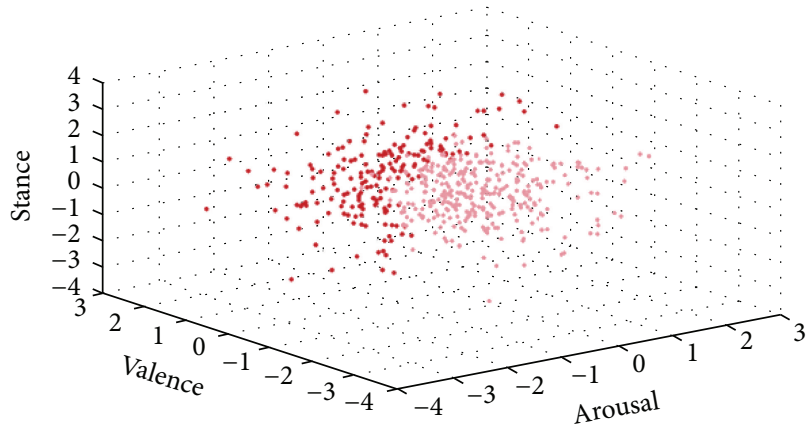

Figure 3: Two kinds of negative emotional distribution after the cognitive reappraisal.

space for making the robot more lively and anthropopathic. So $t$ 's value range is a nonnegative real number, and $S_{t}$ is a real number point. Considering the influence of input microexpressions from participants, the stimulus emotional space $W=\left\{w_{1}, w_{2}, \ldots, w_{m}\right\}$ is introduced in the robot emotional regulation. Here, $m$ is the number of emotional states contained within the microexpressions. On the basis of Ekman's emotion theory, calming is included in the emotional states space to make the robot more similar to human. So the stimulus emotional state space is

$$
\begin{array}{r}
W=\{\text { anger }, \text { disgust, fear, happiness, } \\
\text { sadness, surprise, calming }\} .
\end{array}
$$

According to the microexpression recognition method in [24], the facial microexpression of participants in the communication is mapped into 7 prototypical emotions in order to simplify the experiment.

\subsection{Emotion Modeling in Active Field}

3.2.1. Emotional States Space in Active Field. Dynamical psychology shows that human psychology also requires energy (namely, psychological energy), as other physical dynamical systems. According to the reactions to external excitations [25-28], in this research, the facial emotion type and the action range correspond to the position of energy source and 
the energy size, respectively. Based on Kismet's emotional space, the concept of the field is introduced into the emotional state space for describing emotional spatiotemporal property and measuring energy change among emotions. To this end, we need consider the following three problems. First, which emotional state is activated in the field? Second, what is the source of the activated emotions? Third, what is the field distribution around them? In our emotional model, the interaction between external stimulus and robot's emotion in the active field forms the emotional state space. Here, the size of field source is determined by the activated intensity of emotional state, and position of field source is determined by emotional category. Field intensity distribution in the emotional state space is determined by the emotional state system which is composed of stimulus states and robot's current emotions. From the field theory, the activated intensity $\vec{E}_{M}$ at any point $M(x, y, z)$ in emotional state space is

$$
\vec{E}_{M}=\sum_{i=1}^{n} \frac{Q_{i}}{\mu r_{i}^{2}} \vec{r}_{i}^{o}
$$

where $i$ is the number of emotional sources, $r_{i}$ is the distance from emotional source $i$ to point $M(x, y, z), \vec{r}_{i}^{0}$ is $r_{i}$ 's direction vector, $\mu$ is a coefficient, and $Q_{i}$ is the intensity of emotional source $i$.

The emotional vector field corresponds to the scalar potential field. The emotional activated intensity $\vec{E}$ describes the field in the space from the force perspective. Accordingly, robot emotional state transforms from current to next via the work done by the active field. On the other hand, the emotional potential $\varepsilon$ describes the field by energy, so the emotional potential energy is represented by $\varepsilon$ 's value which is only determined by field sources. $\varepsilon$ 's value is equal to, in numeral, emotional potential energy of the unitage. The computing method about emotional potential energy $M(x, y, z)$ having $n$ activated emotional states is (Figure 4)

$$
\begin{aligned}
\varepsilon_{M}(x, y, z) & =\sum_{i=1}^{n} \frac{Q_{i}}{\mu r_{i}} \\
& =\sum_{i=1}^{n} \frac{Q_{i}}{\mu \sqrt{\left(x-x_{i}\right)^{2}+\left(y-y_{i}\right)^{2}+\left(z-z_{i}\right)^{2}}} .
\end{aligned}
$$

3.2.2. State Transition Probability for Emotions. Individual emotional state is driven and produced by psychological energy. In the active field state space, the family of next emotional states is chosen by the potential energy generated by the stimulus and robot's current emotion. The greater the emotional potential energy the point possesses, the more the probability of this potential surface the next emotional state has. Emotional activation threshold could effectively solve the problem of emotional over sensitivity and overflow. When the emotional potential energy is in a certain interval $[a, b]$, this emotional state might be activated. And in other cases, emotions do not have activated probability.

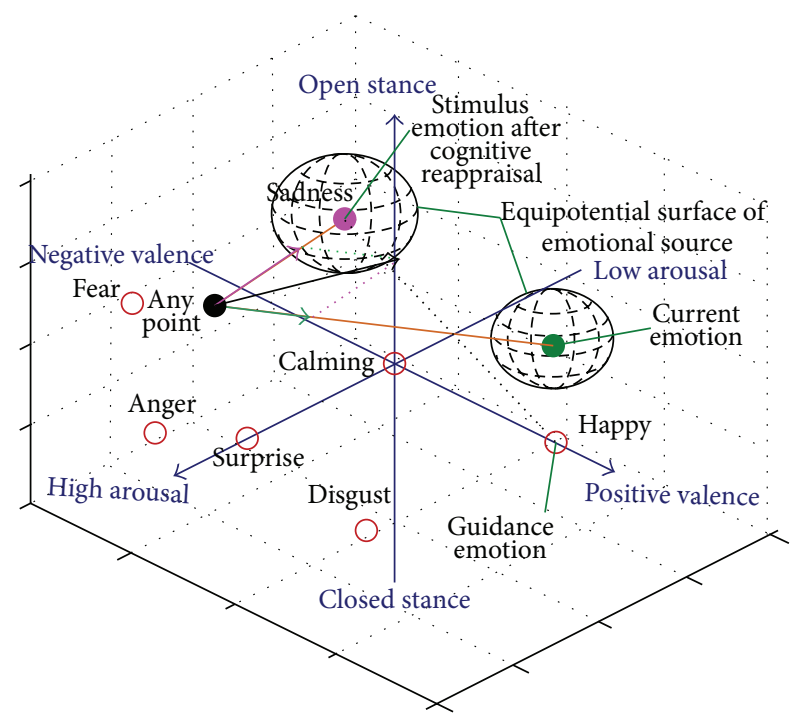

FIgURE 4: The current and stimulus emotions influence any point in active field state space.

In $[a, b]$, the sum of emotional potential energy for each point along the field direction is

$$
\varepsilon_{\text {sum }}=\int_{a}^{b} \varepsilon(x, y, z) d s .
$$

The transition probability from the current emotional family $i$ to the next $j$ is

$$
P_{i j}=\frac{\varepsilon_{j}}{\varepsilon_{\text {sum }}} .
$$

3.3. Emotional Regulation Based on HMM. Human emotional regulation can be divided into two steps; the first step is the basis of cognitive reappraisal and the second is correlated with personality factor. So this paper regards emotional regulation process as a double stochastic process, and the first one could not be directly observed. In other words, the emotional regulation process can be imitated by a hidden Markov model (HMM) as shown in Figure 5.

In the hidden process, $\varepsilon_{t}$ is the psychological energy of emotional family $S_{t}^{\prime}$ at time $t$, and $t$ is a nonnegative real number. After the external stimulus, emotional regulation can be considered continuous in time and space, and it satisfies the following criteria. (1) When the external stimulus occurs, $\Pi$ is initial probability, and it is for final state in the last regulation process to decide. (2) For any $s_{1} \leq t_{1} \leq s_{2} \leq$ $t_{2} \cdots \leq s_{n} \leq t_{n}$, random variables $\varepsilon_{t_{1}}-\varepsilon_{s_{1}}, \varepsilon_{t_{2}}-\varepsilon_{s_{2}}, \ldots, \varepsilon_{t_{n}}-\varepsilon_{s_{n}}$ are independent for each other. (3) For any $s<t$, random variable $\varepsilon_{t}-\varepsilon_{s}$ obeys the probability distribution of formula (7). Here emotional state is $j$ at time $s$, and emotional state is $j$ at time $j$. (4) Emotional regulation process is continuous; namely, $t \rightarrow \varepsilon_{t}$ is a continuous function of time $t$. Emotional states containing with equal psychology energy have the same transition probability, and they are perceived as an emotional family. In fact, this Markov process expounds the transformation among the emotional families. 
TABLE 1: 26 possible directions of emotional sates.

\begin{tabular}{ccccccccccccccccccccccccccccccccccccccccccc}
\hline 1 & 2 & 3 & 4 & 5 & 6 & 7 & 8 & 9 & 10 & 11 & 12 & 13 & 14 & 15 & 16 & 17 & 18 & 19 & 20 & 21 & 22 & 23 & 24 & 25 & 26 \\
\hline$\theta$ & 0 & $\frac{\pi}{4}$ & $\frac{\pi}{2}$ & $\frac{3 \pi}{4}$ & $\pi$ & $-\frac{\pi}{4}$ & $-\frac{\pi}{2}$ & $-\frac{3 \pi}{4}$ & 0 & 0 & $\frac{\pi}{4}$ & $\frac{\pi}{2}$ & $\frac{3 \pi}{4}$ & $\pi$ & $-\frac{\pi}{4}$ & $-\frac{\pi}{2}$ & $-\frac{3 \pi}{4}$ & 0 & 0 & $\frac{\pi}{4}$ & $\frac{\pi}{2}$ & $\frac{3 \pi}{4}$ & $\pi$ & $-\frac{\pi}{4}$ & $-\frac{\pi}{2}$ & $-\frac{3 \pi}{4}$ \\
\hline$\varphi$ & 0 & 0 & 0 & 0 & 0 & 0 & 0 & 0 & $\frac{\pi}{2}$ & $\frac{\pi}{4}$ & $\frac{\pi}{4}$ & $\frac{\pi}{4}$ & $\frac{\pi}{4}$ & $\frac{\pi}{4}$ & $\frac{\pi}{4}$ & $\frac{\pi}{4}$ & $\frac{\pi}{4}$ & $-\frac{\pi}{2}$ & $-\frac{\pi}{4}$ & $-\frac{\pi}{4}$ & $-\frac{\pi}{4}$ & $-\frac{\pi}{4}$ & $-\frac{\pi}{4}$ & $-\frac{\pi}{4}$ & $-\frac{\pi}{4}$ & $-\frac{\pi}{4}$ \\
\hline
\end{tabular}

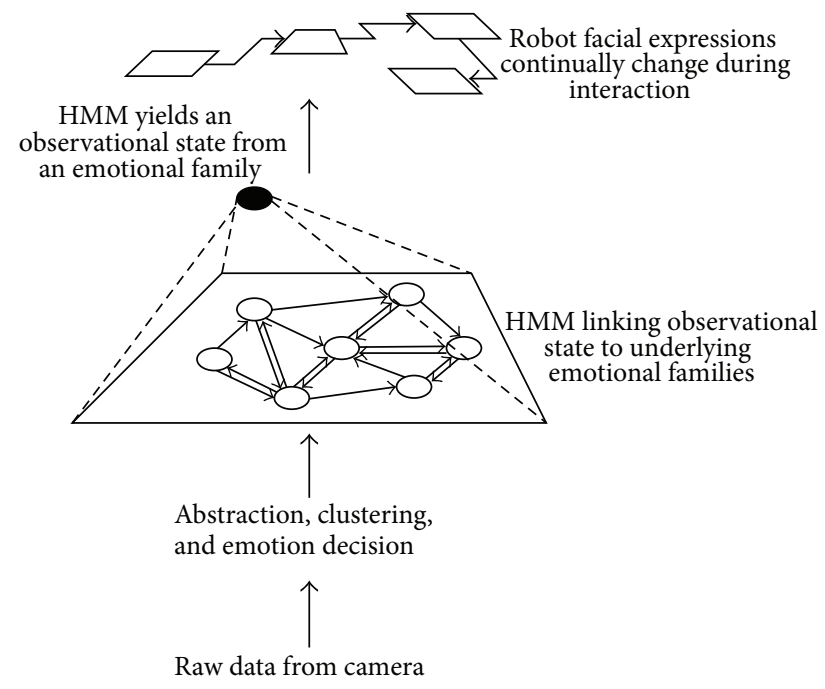

FIgURE 5: Emotional regulation based on HMM.

Another stochastic process is used for outputting a specific emotional state, and this state can be expressed by expression, language, behavior, and so forth. However, these symbols all are emotional extrinsic manifestations, and the emotional state is the motivation to them. We have to both consider the emotional diversity and the system real-time, so there are 26 possibilities in each emotional family (namely, 26 emotional states) as shown in Table 1 . The angle with the high arousal axis is $\theta$, and $-\pi \leq \theta \leq \pi$. The angle with the arousal-valence plane is $\varphi$, and $-\pi / 2 \leq \varphi \leq \pi / 2$. And the angle between possible direction $v$ and the linkage between robot's current emotion and stimulus state is defined as $\sigma_{\nu}$. Emotional state transition probability $P_{\nu}^{\prime}$ is decided by the angle $\sigma_{\gamma}$. When the external stimulus is more than one, angle $\sigma_{\nu}$ to different stimulus may be equal. In this case, the next emotion will randomly select among them

$$
P_{\nu}^{\prime}=\frac{\pi-\sigma_{v}}{n \pi-\sum_{\nu=1}^{n} \sigma_{\nu}}, \quad n=26 .
$$

\section{Emotional Robot}

The robot mechanical structure and shape imitate cartoon. As shown in Figure 6, there are 10 degrees of freedom in the head and arms and more than 100 kinds of facial expressions. So the robot could express emotions with arms, head, and even facial expressions. The robot's upper-body motion mechanism shown in Figure 7 is made up of 10 DC motors and some adapting piece. In the interaction, robot

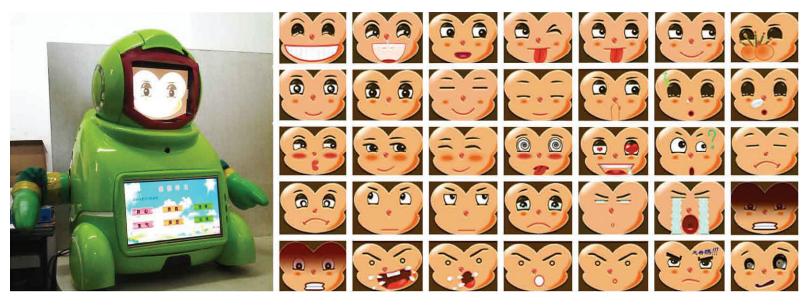

FIGURE 6: Robot and its representative expressions.

emotion will trigger a series of external performances like facial expressions, head gestures, arm actions, and so on. The behavior expression rule is as follows.

IF emotional state THEN robot performances.

Here, the type of performances is closely related to the emotional family and there are some slight differences in the specific emotional state. The range is proportional to the emotional intensity (namely, the emotion potential energy). So the robot vivid behavior is a hybrid between the emotional type and active degree and could realistically express the robot internal emotional changing.

\section{Experiment}

5.1. Emotional Cognitive and Psychological Energy. As discussed in Section 1, the discrete model for emotional classification and regulation is vital limitations. So for expanding the robot emotional output information to continuous space, we use one of the most influential AVS 3D models in the field of affective computing domain: that proposed by Breazeal [20]. Based on Gross reappraisal strategy explained in Section 2.1, we describe the cognitive emotional model and this methodology in the AVS space. The emotional coordinates were calculated on the basis of volunteers' responses to the emotional state along three emotional dimensions of arousal, valence, and stance. And all emotional dimensions are bounded within a range of $[-10,10]$ and the "calming" state is placed on the original point $(0,0,0)$.

Figure 8 graphically shows an example of psychological energy distribution in the active field state space, where both the current emotional state and the stimulus emotional state after cognitive reappraisal as psychological energy resources are trying to influence emotional regulation process. At the moment, the coordinate values of robot's own emotional state are $(5,5,0)$, and external stimulus derived from microexpression is sadness whose coordinate value is $(-6,-4,0)$. Robot physiological endurance coefficient $\chi=0.6$. As can be seen from Figure 8 the emotional energy gradually 

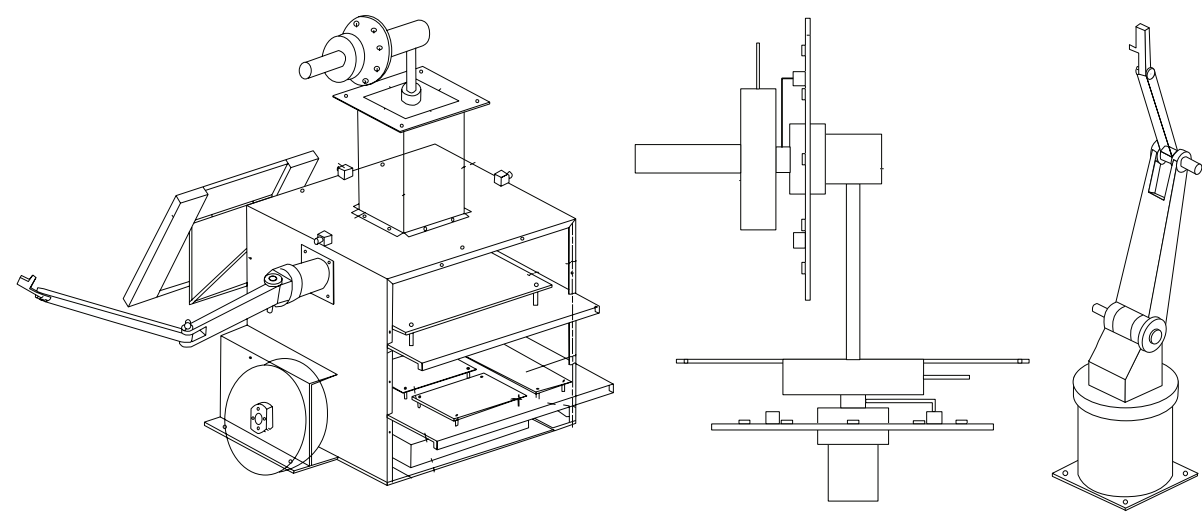

FIGURE 7: Robot mechanical structure.

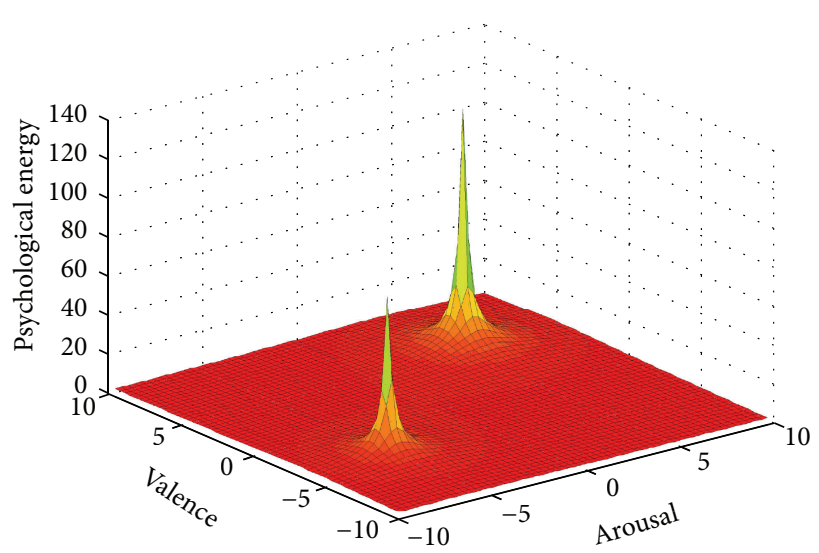

Figure 8: Psychological energy distribution caused by cognitive stimulus and robot's current emotional state.

dies down along with the increase of distance between the emotional state and the emotional source. And psychological energy rapidly declines round the emotional sources. So we set a pair of threshold to limit emotional activation scope. If the energy of emotional states is greater or less than the threshold, these emotional states are impossible to happen. This phenomenon is entirely consistent with classical emotional theory in the field of psychology [29]. Based on the relative positions of the next emotional state and emotional source, that the emotional family contains higher energy will has greater transition probability than the emotional family which contains lower energy. From this, the transition probability in the first stochastic process of HMM can be figured out.

5.2. Emotional Regulation Process. According to Section 3.3, the distribution of emotional family's probability is calculated under a pair of emotions, robot's cognitive stimulus emotional state and its own current emotional state, and then the output of robot emotional state has 26 kinds of possibility on the basis of the spatial relationship among the current emotion, stimulus emotion, and next emotion. This method highlights the capability to find a large amount of intermediate emotional states, which are extremely vital since

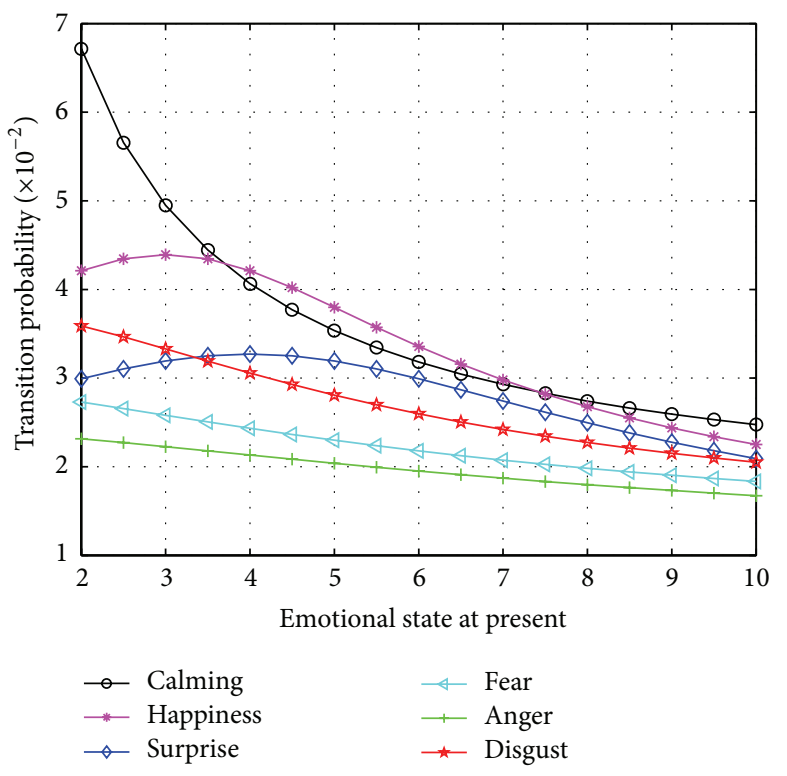

FIGURE 9: Example of emotional family's probability.

they enrich the output of the robot emotional regulation system. In Figure 9, external stimulus emotional state coming from microexpression is "sadness," and we can observe the transition probability's microvariation of emotional families where 6 prototypical emotions (except sadness, because the psychological energy of sadness exceeds the threshold value, and it will not happen) are with robot's own emotional state changes. Here, robot's own emotion is located at any point arousal $=$ valence $\in[2,10]$ and stance $=0$.

Figure 10 shows the robot emotional regulation process with calming initial emotional state. In $0-15 \mathrm{~s}$, robot's own emotion remained about the same under no external stimulus. At the $15 \mathrm{~s}$, an external stimulus "disgust" derived from microexpression occurred, so robot emotion's negative degree gradually increased during $15-35 s$ and achieved the balance around the $35 \mathrm{~s}$. Then this emotional experience was with the robot for about $15 \mathrm{~s}$. Because the "disgust" stimulus has disappeared a while, robot emotional state gradually trended to "calming" during 50-65s [30]. At the $65 \mathrm{~s}$, an 


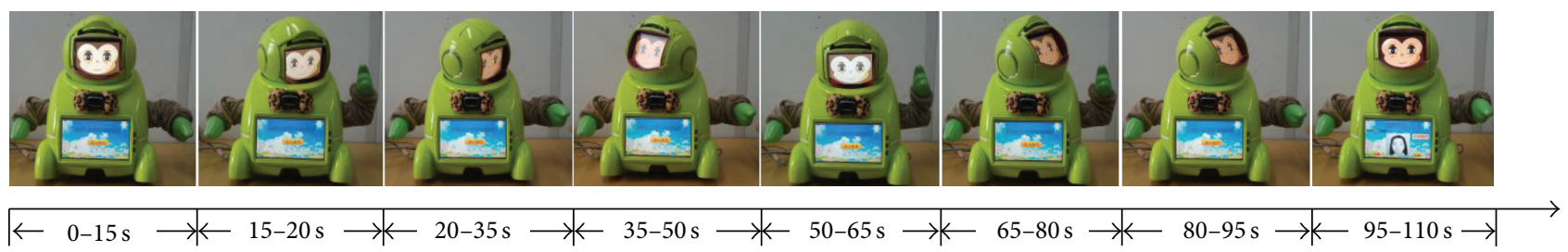

FIGURE 10: Robot emotional regulation process with different external stimulus.

external stimulus "happiness" derived from microexpression occurred, so robot emotion's positive degree gradually increased during 65-80 s and achieved the balance around the $80 \mathrm{~s}$. Then similar emotional experience was with the robot for about $15 \mathrm{~s}$ and gradually waned during $95 \mathrm{~s}$.

Filtered 34 volunteers participate in the human-robot interactions and each person experiments 100-time interaction in accordance with specified criteria. Each participant fills in the predictive scale before the experiment started. In this scale, participants forecast robot's next output state by the simplified affect scale (as Figure 2) including 7 typical stimulus states and 7 typical own emotional sates (a total of $7 \times 7$ kinds of possible typical inputs). The participant fills in the satisfaction survey for each interaction during the interaction. This survey designs two options (agreement and disagreement) for each interaction. From the evaluation results, if they reason out robot's emotional output in advance, the average matching rate is $69.75 \%$ after experience. However, it rises to $97.52 \%$ when only considering participants' agreement to robot output emotions. Objectively speaking, the use of the emotion model based on the cognitive reappraisal in active field allows robot to imitate the hominine emotional regulation, and that is, in fact, the aim of our work. But the obtained results are difficult to compare with other emotional regulation studies that can be found in literature, because most of such studies do not recognize stimulus emotions in microexpressions and transfer emotional states in arousal-valence-stance terms. Moreover the few studies that do have not been tested under physical robot experimental conditions (specific robot device and experimental platform refer to $[30,31])$ and do not provide as output the coordinates of the studied emotional state in the $3 \mathrm{D}$ space.

\section{Conclusion}

In this paper, the noteworthy feature of emotional regulation work was out of the simply interactive mode providing the classification and jump in terms of a set of emotional labels, and it operated in a 3D emotional space enabling a wide range of intermediary emotional states obtained under the external stimulus. Moreover, this system focused on the research field of emotional regulation depending on natural facial expression cognition and proposed a microexpression cognition and emotional regulation model based on Gross reappraisal strategy. Gross cognitive reappraisal strategy effectively decreased negative emotional experience and behavioral expression, so it could provide an intelligent cognition style to computer/robot acting as a positive role in
HCI. HMM double stochastic process makes robot emotions have more diversification in human-robot interaction. In general, the use of HMM emotional regulation model based on cognitive reappraisal in active field allows robot to imitate the hominine emotional regulation naturally.

Our current research only proposed a computable emotion model applied to universal psychological significance in continuous space but not considered with specific emotion changes. Following from this, future works should be oriented to the study of nature inspired cognitive-affective computing by means of emotion modeling in continuous active space and especially need pay more attention to the multimodal external stimulus and the pervasive emotion computing.

\section{Conflict of Interests}

The authors declare that there is no conflict of interests regarding the publication of this paper.

\section{Acknowledgments}

This work is supported by National Natural Science Foundation of China (no. 61432004, 61170115), National Key Technologies R\&D Program of China (no. 2014BAF08B04), and the Foundation of Beijing Engineering and Technology Center for Convergence Networks and Ubiquitous Services.

\section{References}

[1] S. S. Tompkins, Affect Imagery Consciousness: Volume 1: The Positive Affects, Springer, London, UK, 1962.

[2] C. Izard, The Face of Emotion, vol. 23, Appleton-Century-Crofts, New York, NY, USA, 1971.

[3] G. Valenza, A. Lanata, and E. P. Scilingo, "The role of nonlinear dynamics in affective valence and arousal recognition," IEEE Transactions on Affective Computing, vol. 3, no. 2, pp. 237-249, 2012.

[4] R. S. Lazarus, "Relational meaning and discrete emotions," in Appraisal Processes in Emotion: Theory, Methods, Research, pp. 37-67, Oxford University Press, New York, NY, USA, 2001.

[5] P. Ekman, Lie Catching and Microexpressions, Oxford Universit Press, 2009.

[6] L. Cañamero, "Modeling motivations and emotions as a basis for intelligent behavior," in Proceedings of the 1st international Conference on Autonomous Agents (AGENTS '97), pp. 148-155, 1997. 
[7] S. Gadanho, "Reinforcement learning in autonomous robots: an empirical investigation of the role of emotions," in Emotions in Human and Artifacts, MIT Press, 2002.

[8] J. Velásquez, "An emotion-based approach to robotics," in Proceedings of the IEEE/RSJ International Conference on Intelligent Robots and Systems (IROS '99), vol. 1, pp. 235-240, Kyongju, Republic of Korea, October 1999.

[9] R. R. Murphy, C. L. Lisetti, R. Tardif, L. Irish, and A. Gage, "Emotion-based control of cooperating heterogeneous mobile robots," IEEE Transactions on Robotics and Automation, vol. 18, no. 5, pp. 744-757, 2002.

[10] W. Burgstaller, R. Lang, P. Porscht, and R. Velik, “Technical model for basic and complex emotions," in Proceedings of the 5th IEEE International Conference on Industrial Informatics, pp. 1007-1012, 2007.

[11] W. Wundt, Principles of Physiological Psychology, Macmillan Press, New York, NY, USA, 1910.

[12] H. Schlosberg, "Three dimensions of emotion," Psychological Review, vol. 61, no. 2, pp. 81-88, 1954.

[13] P. J. Lang, M. M. Bradley, and B. N. Cuthbert, "Emotion, motivation, and anxiety: brain mechanisms and psychophysiology," Biological Psychiatry, vol. 44, no. 12, pp. 1248-1263, 1998.

[14] C. Osgood, The Measurement of Meaning, University of Illinois Press, 1975.

[15] J. Panksepp, Affective Neuroscience: The Foundations of Human and Animal Emotions, Oxford University Press, New York, NY, USA, 2004.

[16] J. A. Russell and A. Mehrabian, "Evidence for a three-factor theory of emotions," Journal of Research in Personality, vol. 11, no. 3, pp. 273-294, 1977.

[17] K. Scherer and P. Ekam, Approaches to Emotions, Lawrence Erlbaum Associates, 1984.

[18] A. Ortony, G. L. Clore, and A. Collins, The Cognitive Structure of Emotions, Cambridge University Press, London, UK, 1988.

[19] M. Zecca, S. Roccella, M. C. Carrozza et al., "On the development of the emotion expression humanoid robot WE-4RII with RCH-1," in Proceedings of the 4th IEEE-RAS International Conference on Humanoid Robots, pp. 235-252, Tokyo, Japan, November 2004.

[20] C. Breazeal, "Function meets style: insights from emotion theory applied to HRI," IEEE Transactions on Systems, Man and Cybernetics Part C: Applications and Reviews, vol. 34, no. 2, pp. 187-194, 2004.

[21] H. Yang, Z. Pan, and G. Liu, "Comprehensive computational model of emotions," Journal of Computer Research and Development, vol. 45, no. 4, pp. 579-587, 2008.

[22] W. H. Kim, J. W. Park, W. H. Lee, and M. J. Chung, "Stochastic approach on a simplified OCC model for uncertainty and believability, in Proceedings of the IEEE International Symposium on Computational Intelligence in Robotics and Automation (CIRA '09), pp. 66-71, Daejeon, Republic of Korea, December 2009.

[23] J. J. Gross, "Emotion regulation: affective, cognitive, and social consequences," Psychophysiology, vol. 39, no. 3, pp. 281-291, 2002.

[24] S.-J. Wang, H.-L. Chen, W.-J. Yan, Y.-H. Chen, and X. Fu, "Face recognition and micro-expression recognition based on discriminant tensor subspace analysis plus extreme learning machine," Neural Processing Letters, vol. 39, no. 1, pp. 25-43, 2014.
[25] J. Zhang, X. Wang, and H. Xie, "Phonon energy inversion in graphene during transient thermal transport," Physics Letters, $A$, vol. 377, no. 9, pp. 721-726, 2013.

[26] Q. Xiong, B. Li, J. Xu, X. Wang, L. Wang, and W. Ge, "Efficient 3D DNS of gas-solid flows on Fermi GPGPU," Computers and Fluids, vol. 70, pp. 86-94, 2012.

[27] Q. Xiong, E. Madadi-Kandjani, and G. Lorenzini, "A LBMDEM solver for fast discrete particle simulation of particle-fluid flows," Continuum Mechanics and Thermodynamics, vol. 26, no. 6, pp. 907-917, 2014.

[28] J. Zhang, Y. Wang, and X. Wang, "Rough contact is not always bad for interfacial energy coupling," Nanoscale, vol. 5, no. 23, pp. 11598-11603, 2013.

[29] M. A. Salichs and M. Malfaz, "A new approach to modeling emotions and their use on a decision-making system for artificial agents," IEEE Transactions on Affective Computing, vol. 3, no. 1, pp. 56-68, 2012.

[30] L. Xin, X. Lun, W. Zhi-Liang, and F. Dong-Mei, "Robot emotion and performance regulation based on HMM," International Journal of Advanced Robotic Systems, vol. 10, article 160, 2013.

[31] P. Xiaolan, X. Lun, L. Xin, and W. Zhiliang, "Emotional state transition model based on stimulus and personality characteristics," China Communications, vol. 10, no. 6, pp. 146-155, 2013. 


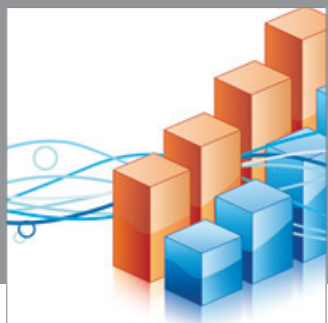

Advances in

Operations Research

mansans

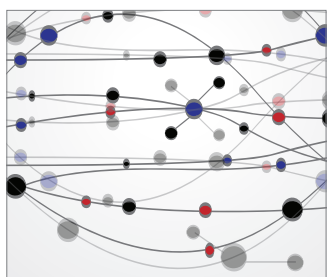

The Scientific World Journal
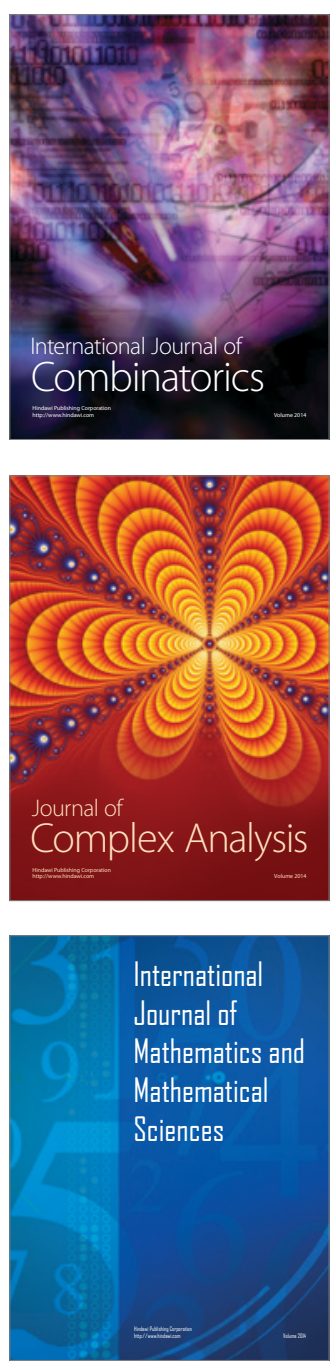
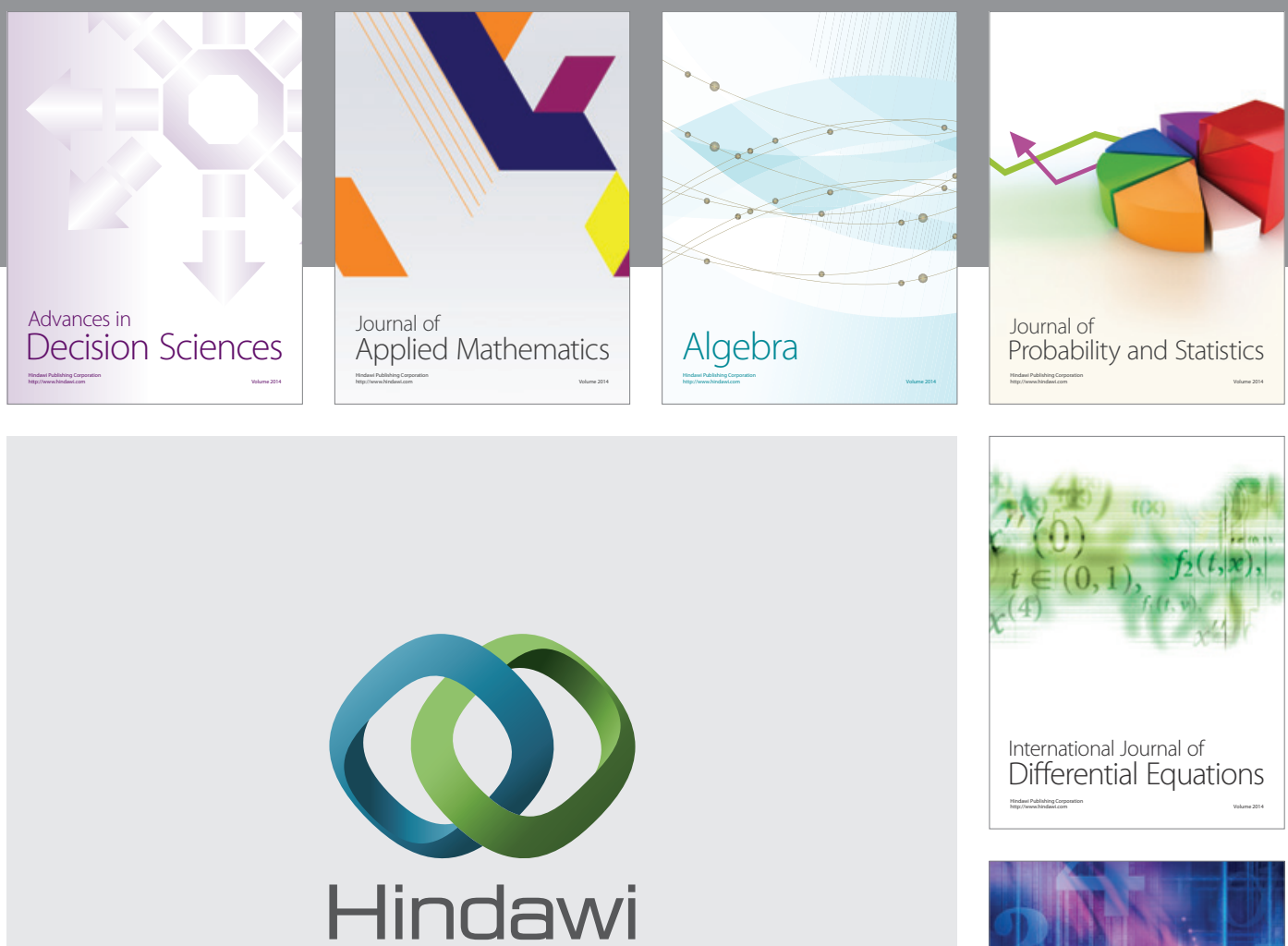

Submit your manuscripts at http://www.hindawi.com
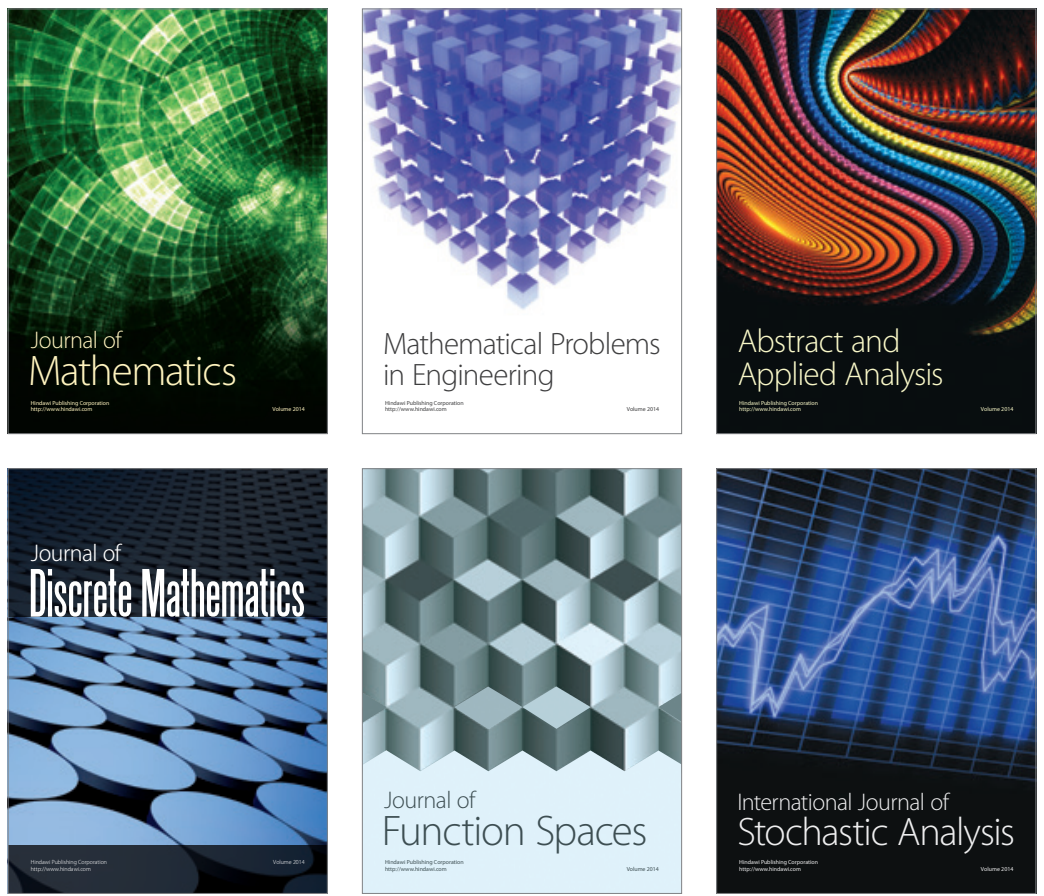

Journal of

Function Spaces

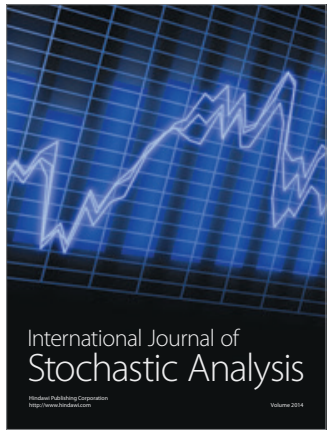

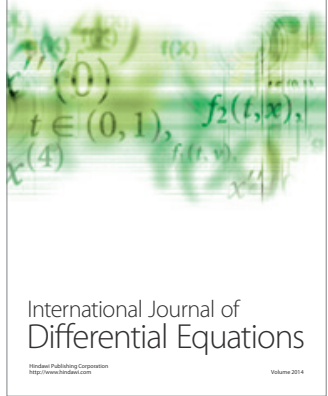
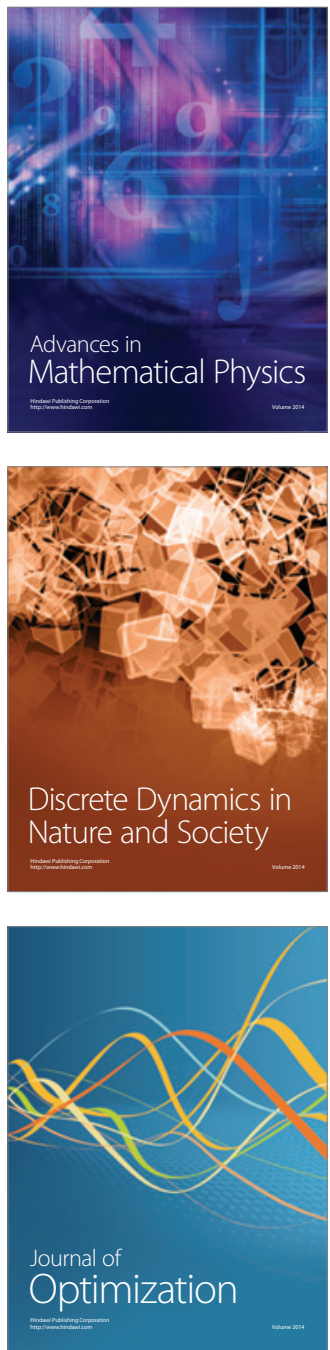